\title{
Retinaculotomía endoscópica de doble portal en el síndrome del túnel carpiano
}

\author{
Álvaro C. Ojeda, Víctor A. Contreras, Edgardo S. Sanzana \\ Departamento de Cirugía, Facultad de Medicina, Universidad de Concepción, Chile
}

\section{RESUMEN}

Objetivo: Evaluar los resultados de la retinaculotomía endoscópica para tratar el síndrome del túnel carpiano mediante la técnica de doble portal de Chow, entre enero de 2006 y diciembre de 2015. Materiales y Métodos: Estudio de 179 pacientes (edad promedio 48.2 años [rango 32-68]), con 217 casos de síndrome del túnel carpiano idiopático y un seguimiento promedio de 97.9 meses. Los pacientes eran 145 mujeres (81\%) (31 bilaterales) y 34 hombres (19\%) (7 bilaterales) y fueron evaluados con la Symptom Severity Scale (SSS) y la Functional Status Scale (FSS) del Boston Carpal Tunnel Questionnaire (BCTQ). Resultados: El puntaje medio de la SSS-BCTQ fue de 3,20 $\pm 0,26$ antes de la cirugía, mejoró a 1,30 $\pm 0,12$ a los 6 meses y se mantuvo en 1,25 $\pm 0,11$ a largo plazo. El puntaje medio de la FSS-BCTQ fue de $2,57 \pm 0,29$ antes de la cirugía, mejoró a 1,28 $\pm 0,18$ a los 6 meses y se mantuvo en $1,20 \pm 0,09$ a largo plazo. Hubo 7 casos $(3,2 \%)$ de neuropraxia posquirúrgica transitoria. No hubo conversiones a técnica abierta. Conclusión: La liberación endoscópica del túnel carpiano con la técnica de Chow es un método quirúrgico eficaz y seguro para tratar el síndrome del túnel carpiano idiopático.

Palabras clave: Síndrome del túnel carpiano; técnica de doble portal de Chow; liberación endoscópica; compresión neural; nervio mediano.

Nivel de Evidencia: III

\section{Dual-portal endoscopic release in carpal tunnel syndrome}

\section{ABSTRACT}

Objective: To evaluate the outcomes of endoscopic release of the transverse carpal ligament (TCL) in carpal tunnel syndrome (CTS) using the Chow dual-portal technique between January 2006 and December 2015. Materials and Methods: Study population consisted of 217 cases of idiopathic CTS, in 179 patients, 145 females (81\%) (31 bilateral cases) and 34 males (19\%) (7 bilateral cases), with an average age of 48.2 years (range, 32-68) and an average follow-up of 97.9 months. The symptom severity and functional evaluations were performed using the Boston Carpal Tunnel Questionnaire Symptoms Severity Scale (BCTQ-SSS) and the Functional Status Scale (BCTQ-FSS). Results: The average BCTQ-SSS was $3.20 \pm 0.26$ in the preoperative period, which improved to $1.30 \pm 0.12$ at the 6 -month postoperative follow-up and remained at $1.25 \pm 0.11$ in the long-term. The average BCTQ-FSS was $2.57 \pm 0.29$ in the preoperative period, which improved to $1.28 \pm 0.12$ at the 6-month postoperative follow-up and remained at $1.20 \pm 0.09$ in the long-term. There were 7 cases $(3.2 \%)$ of transient postoperative neurapraxia. No patient required to be converted to open technique. Conclusion: The endoscopic carpal tunnel release with Chow technique is an effective and safe surgical method for the treatment of idiopathic CTS.

Key words: Carpal tunnel syndrome; Chow dual-portal technique; endoscopic release; neural compression; median nerve.

Level of Evidence: III

\section{INTRODUCCIÓN}

El síndrome del túnel carpiano (STC) es la neuropatía periférica compresiva más frecuente y puede afectar hasta el 3\% de la población general, y su prevalencia es más alta en el sexo femenino. ${ }^{1}$ Este cuadro fue descrito, por primera vez, por Paget, en 1854 y el reporte más antiguo de su resolución quirúrgica fue publicado por Learmonth y data de 1933. ${ }^{2,3}$ Desde entonces, todas las técnicas quirúrgicas empleadas para tratar el STC buscan la descompresión del nervio mediano mediante la sección del retináculo flexor (RF) del carpo.

Recibido el 23-2-2020. Aceptado luego de la evaluación el 5-5-2020 • Dr. EDGARDO S. SANZANA • esanzana@udec.cl ID https://orcid.org/0000-0001-6656-435X Cómo citar este artículo: Ojeda ÁC, Contreras VA, Sanzana ES. Retinaculotomía endoscópica de doble portal en el síndrome del túnel carpiano. Rev Asoc Argent Ortop Traumatol 2020;85(4):349-356. https://doi.org/ 10.15417/issn. 1852-7434.2020.85.4.1062 
Durante más de 50 años, la sección abierta del RF resultó un procedimiento quirúrgico eficaz para los pacientes con STC y síntomas persistentes, que no responden al tratamiento conservador. ${ }^{4}$ Sin embargo, esta técnica puede provocar complicaciones, como lesión del ramo palmar cutáneo del nervio mediano, dolor residual en los pilares óseos del túnel carpiano o síntomas irritativos en la cicatriz..$^{5,6}$

Con la finalidad de reducir las complicaciones de la cirugía abierta para el STC, en la década de 1990, comienza el estudio y el desarrollo de procedimientos de liberación endoscópica del RF y, al mismo tiempo, en 1989, se publican la técnica de un portal de Okutsu y la técnica de doble portal de Chow. ${ }^{7,8}$ Ratificando el trabajo de estos pioneros, otros grupos quirúrgicos continuaron participando activamente en el desarrollo de técnicas endoscópicas, tanto de portal único como de doble portal, entre los que se destacan Agee y Brown, quienes, de manera coincidente e independiente, publicaron sus respectivas experiencias durante $1992 .^{9,10}$

La evidencia acumulada ha demostrado que la liberación retinacular endoscópica en el STC tiene una eficacia y una seguridad clínicas, a largo plazo, equivalentes a las de la cirugía abierta; una ventaja comparativa inicial es la menor cuantía del dolor posoperatorio. No obstante, a partir de los tres meses de la intervención quirúrgica, los resultados de la técnica abierta y los procedimientos endoscópicos tienden a igualarse y permanecen sin diferencias significativas en los puntajes de síntomas y el estado funcional a largo plazo. ${ }^{11-16}$

Este estudio tiene como objetivo exponer nuestra experiencia, evaluada mediante un estudio de cohortes histórico, con la descompresión endoscópica del túnel carpiano en pacientes con STC idiopático que fueron operados mediante la técnica de doble portal de Chow. ${ }^{17}$

\section{MATERIALES Y MÉTODOS}

Entre enero de 2006 y diciembre de 2015, 224 pacientes con STC idiopático, sin respuesta al tratamiento conservador fueron sometidos a cirugía mediante la técnica de liberación endoscópica de doble portal de Chow. Se presentan los resultados obtenidos en 179 de ellos que fueron evaluados y controlados a largo plazo.

Se llevó a cabo un estudio de cohortes histórico de 179 pacientes, con 217 casos de STC idiopático; la edad promedio de los pacientes era de 48.2 años (rango 32-68). El grupo de estudio estaba formado por 145 mujeres (81\%) con 176 casos de STC (31 bilaterales) y 34 hombres (19\%) con 41 casos de STC (7 bilaterales). En 104 pacientes, la extremidad afectada era la derecha y, en 37, la izquierda; 38 pacientes tenían compromiso de ambas extremidades. Todos fueron evaluados con ecotomografía y electromiografía antes de la cirugía y los pacientes con STC bilateral fueron operados de ambas extremidades en el mismo acto quirúrgico. ${ }^{14,18}$

Los pacientes recibieron el alta a las seis horas de la cirugía, y fueron evaluados una vez por semana, durante el primer mes, y mensualmente en el primer semestre, y con un control final a largo plazo. La evaluación consistió en el Boston Carpal Tunnel Questionnaire (BCTQ) de Levine, que consta de dos subescalas independientes. La primera corresponde a la Symptom Severity Scale (SSS) que incluye 11 ítems, con un puntaje de 1 a 5, y cuyos resultados se promedian, y la segunda se trata de la Functional Status Scale (FSS) con 8 ítems y un puntaje de 1 y 5, y cuyos resultados se promedian de manera independiente. ${ }^{19}$ En el estudio estadístico, se realizó un análisis descriptivo para cada una de las variables, calculando frecuencias absolutas y relativas para las cualitativas, y empleando la media y la desviación estándar para las cuantitativas.

\section{Técnica quirúrgica}

Se coloca al paciente en decúbito supino, sin manguito hemostático y con la extremidad superior en una mesa de mano. El primer cirujano se sitúa en el lado cubital del paciente y el segundo cirujano, enfrente. El procedimiento endoscópico se efectúa en seco y se requiere contar con el Sistema ECTRA II diseñado por Chow (Smith $\&$ Nephew Endoscopy, Andover, MA, EE.UU.). Este sistema consta de un videoendoscopio corto de $4 \mathrm{~mm}$ y $30^{\circ}$, retractores de Rangell, cánula ranurada, disector romo curvo, obturadores, gancho palpador, soporte de mano y un kit desechable que incluye bisturí anterógrado, bisturí triangular y bisturí retrógrado (Figura 1).

Preparación estándar de la extremidad y dibujo del gancho del hamate, el pisiforme, el tendón del palmar largo y los portales endoscópicos. El portal proximal se establece entre 1 y $2 \mathrm{~cm}$ radiales al borde proximal del pisiforme y el cubital al palmar largo. El portal distal se localiza $1 \mathrm{~cm}$ proximal a la intersección de una línea longitudinal que sigue al tercer espacio y otra transversal alineada con la base del pulgar abducido. Se infiltra lidocaína al 1,5\% $(3 \mathrm{ml}=45 \mathrm{mg}$ ) en ambos portales. Se agrega sedación mediante infusión continua de propofol al 1\% (FresseniusKabi, Alemania). ${ }^{20}$

La cirugía se inicia con una incisión de $5 \mathrm{~mm}$ en el sitio del portal proximal, luego se identifica el borde proximal del RF, se introduce el disector romo curvo a nivel infrarretinacular del túnel carpiano hasta aflorar a nivel 
del portal distal, instalando la cánula ranurada y el videoendoscopio (Figura 2). Enseguida, es posible visualizar y palpar instrumentalmente el RF, se procede a su sección completa mediante el empleo consecutivo de los bisturíes anterógrado, triangular y retrógrado. Finalmente, se suturan los portales proximal y distal con un punto de piel (Figura 3). ${ }^{17}$

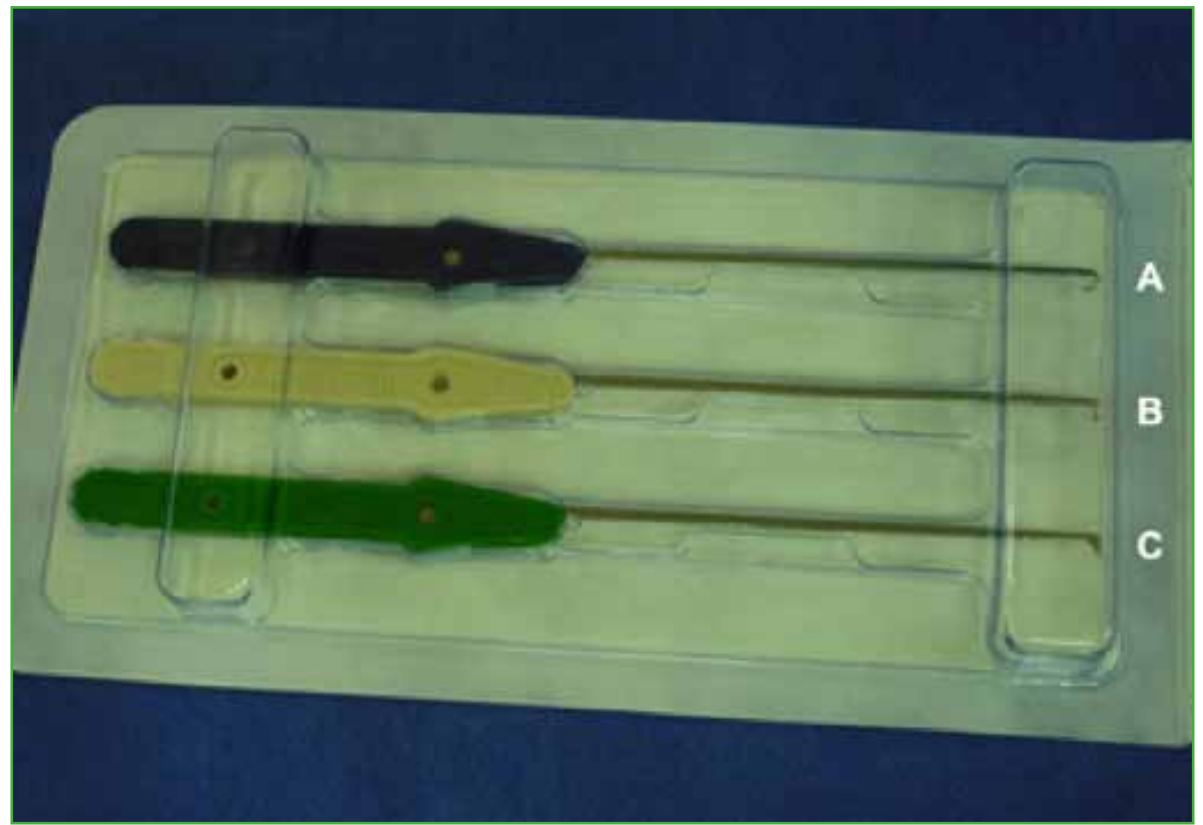

Figura 1. Kit desechable del Sistema ECTRA II de Chow (Smith \& Nephew Endoscopy, Andover, MA, EE.UU.). A. Bisturí retrógrado. B. Bisturí anterógrado. C. Bisturí triangular.
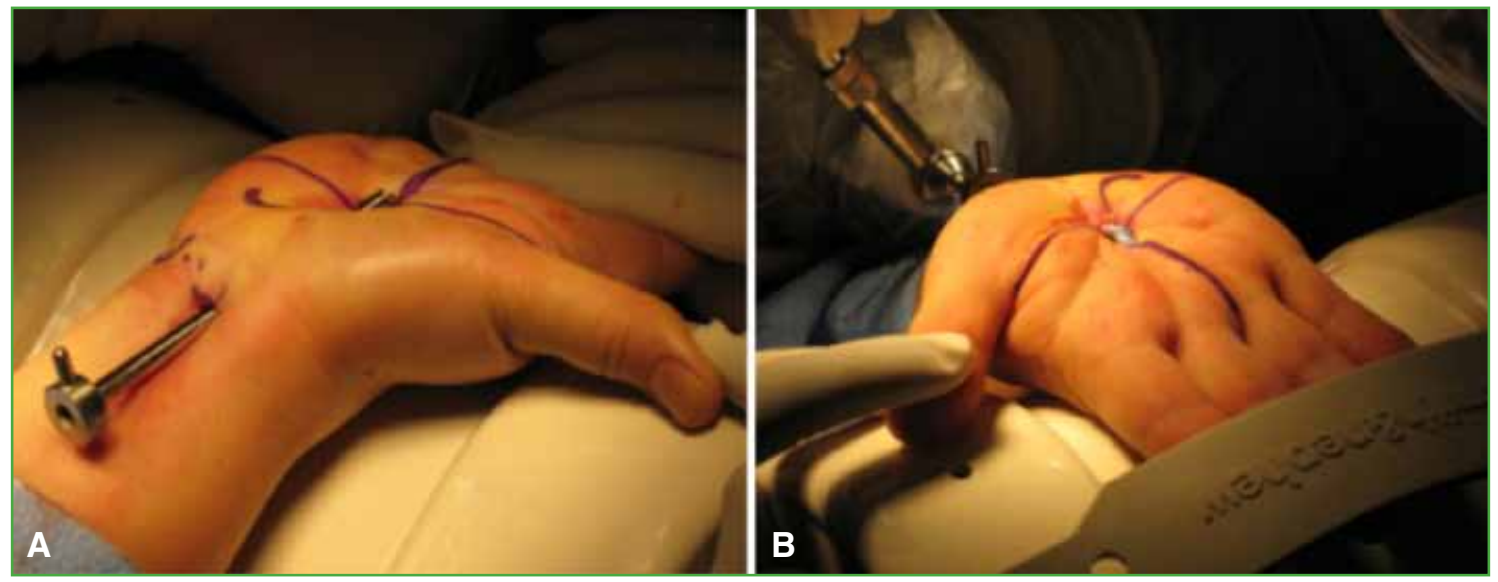

Figura 2. Técnica endoscópica de doble portal de Chow. A. Cánula ranurada localizada en el túnel carpiano.

B. Procedimiento de visualización en seco del retináculo flexor con videoendoscopio corto. 

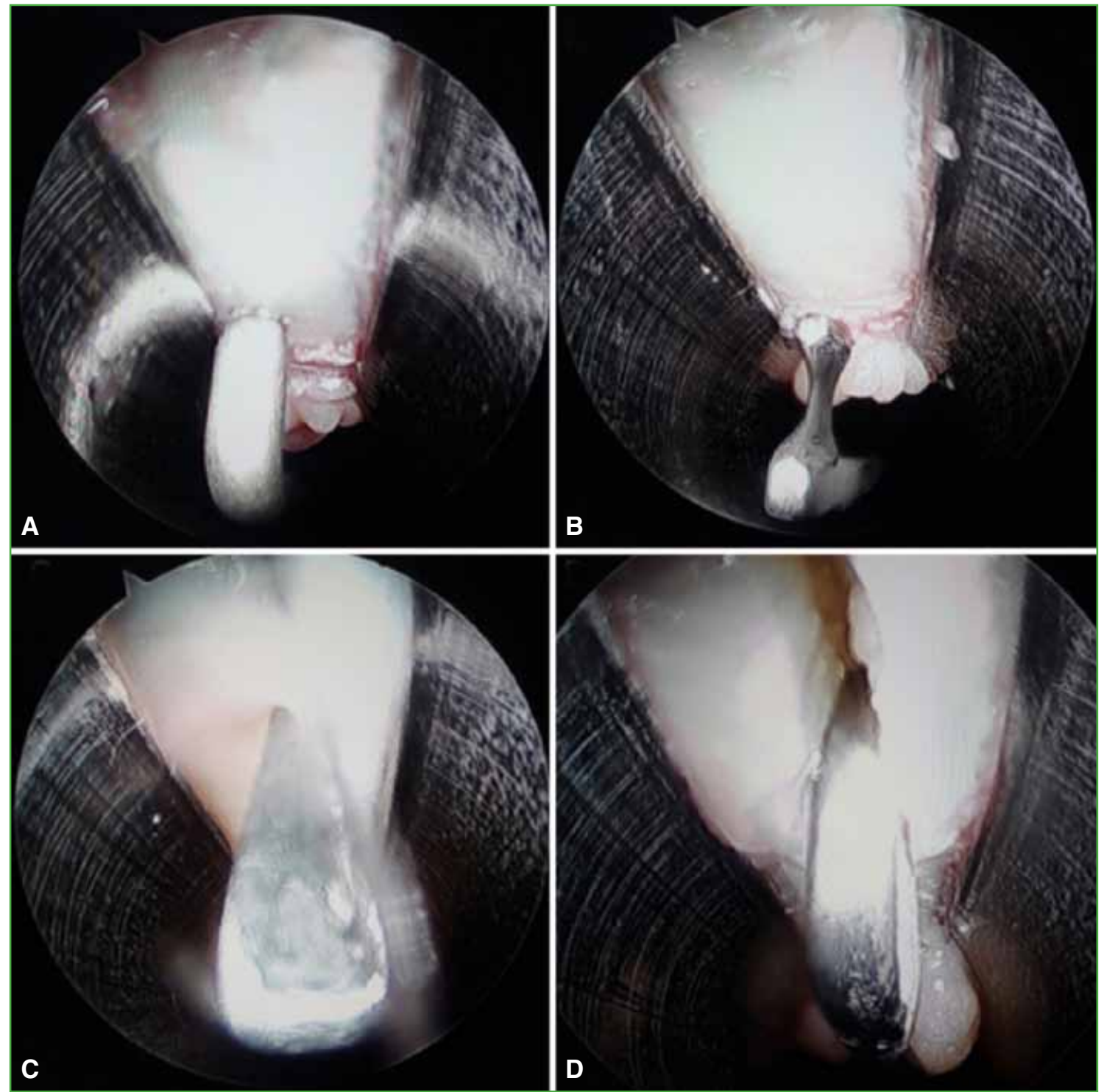

Figura 3. Procedimiento de liberación retinacular endoscópica en el síndrome del túnel carpiano idiopático. A. Gancho palpador a nivel del retináculo flexor. B. Empleo del bisturí anterógrado C. Utilización del bisturí triangular. D. Sección retinacular con bisturí retrógrado.

\section{RESULTADOS}

El tiempo medio de seguimiento fue de 97.9 meses (rango 42-162). Los pacientes refirieron síntomas de STC por un período promedio de 9.8 meses (rango 6-18). Para determinar los resultados, se los evaluó con las escalas del BCTQ, aplicando las subescalas SSS y FSS, antes de la cirugía, a los 6 meses y en el control final a largo plazo.

De esta forma, se determinó que el puntaje medio de la SSS-BCTQ fue de 3,20 \pm 0,26 antes de la cirugía, mejoró con una diferencia estadísticamente significativa a $1,30 \pm 0,12$ a los 6 meses y se mantuvo sin diferencia significativa en 1,25 \pm 0,11 a largo plazo (Figura 4). El puntaje medio de la FSS-BCTQ fue de 2,57 \pm 0,29 antes de la cirugía, mejoró con una diferencia estadísticamente significativa a 1,28 $\pm 0,18$ a los 6 meses y se mantuvo sin diferencia significativa en 1,20 \pm 0,09 a largo plazo (Figura 5). En la evaluación final, el 96\% de los pacientes se manifestó satisfecho con el resultado del procedimiento endoscópico. 


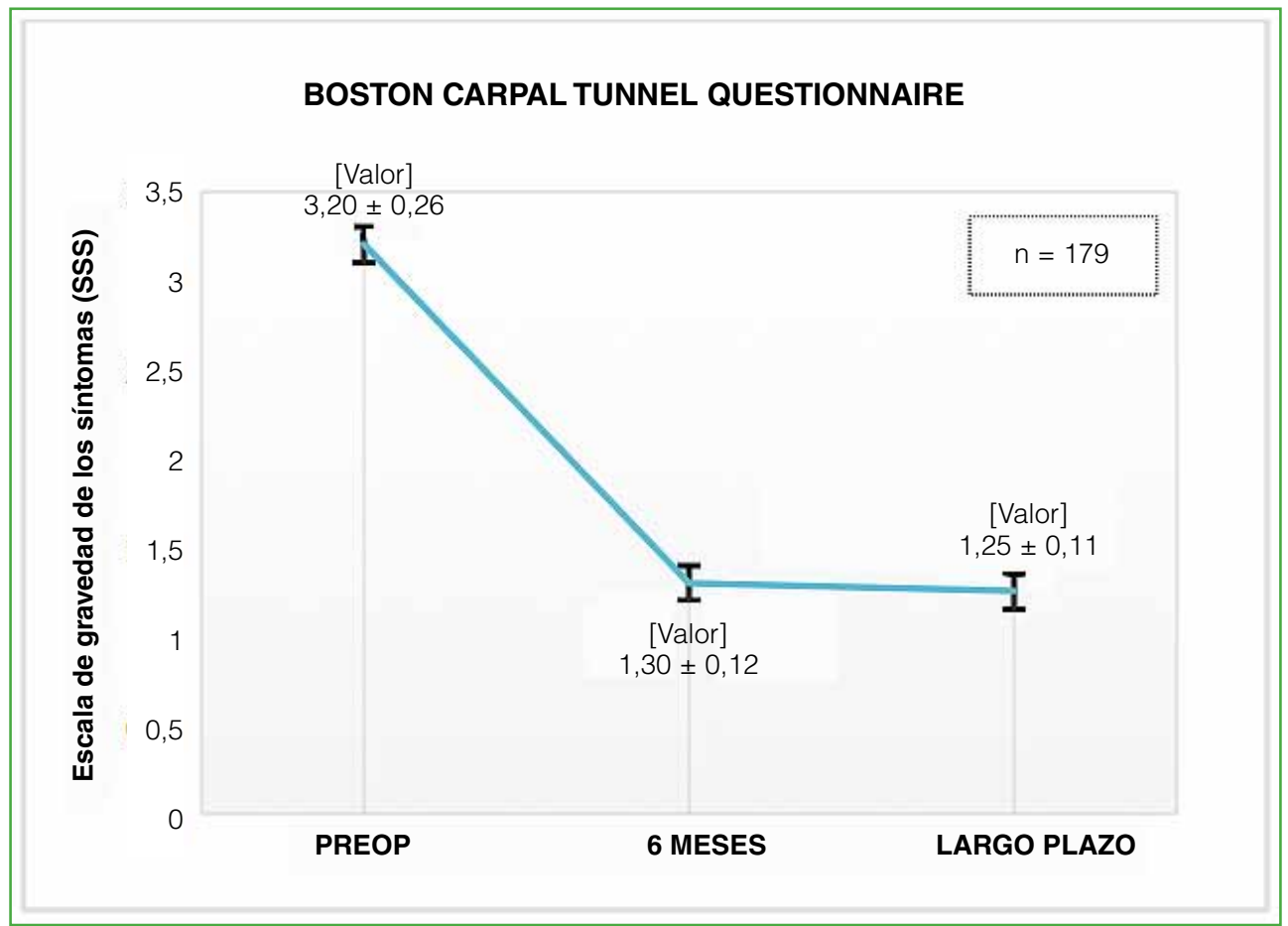

Figura 4. Puntaje medio de la SSS-BCTQ.

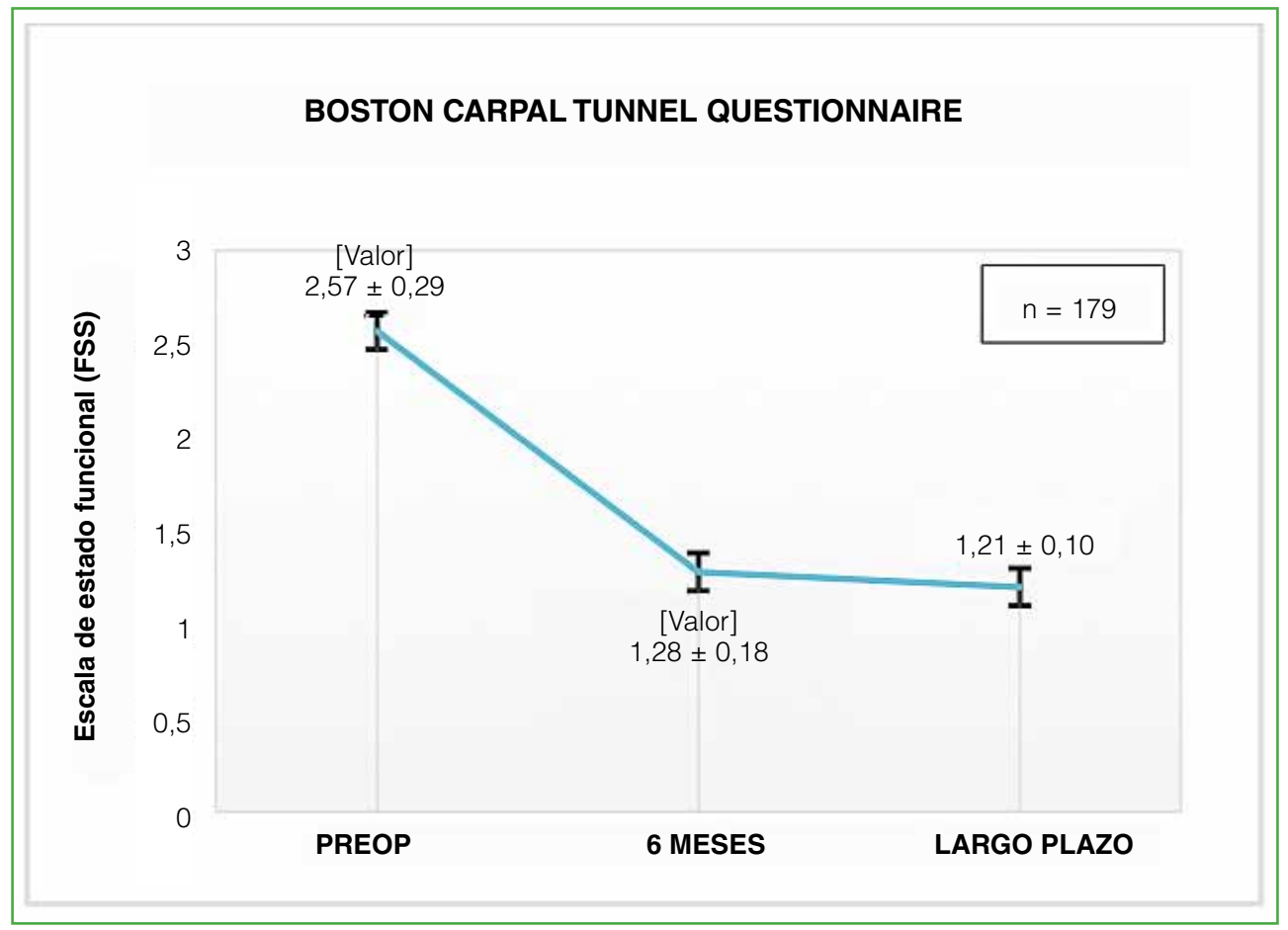

Figura 5. Puntaje medio de la FSS-BCTQ. 
Se registraron siete complicaciones que correspondieron a cuadros de neuropraxia posquirúrgica transitoria $(3,2 \%)$ : tres casos de neuropraxia del nervio digital común del tercer espacio, dos de neuropraxia del nervio cubital y dos de neuropraxia del nervio mediano. Las complicaciones se produjeron en pacientes operados durante los primeros dos años de la década evaluada. Todos estaban recuperados neurológicamente en el control de los 6 meses. No se produjo ninguna complicación neurovascular mayor y no fue necesaria ninguna conversión a técnica abierta.

\section{DISCUSIÓN}

Transcurridos 30 años desde las primeras publicaciones, los procedimientos de liberación retinacular endoscópica del STC han demostrado su eficacia y seguridad, con tasas de éxito que superan el 95\% con el BCTQ de Levine o similares, aunque sin diferencias significativas con la técnica abierta en los resultados a largo plazo. ${ }^{11-16}$ Por lo mismo, es importante destacar que el procedimiento abierto mantiene su validez sobre la base de la sólida ventaja de su simplicidad técnica, aunque sus desventajas siguen siendo las eventuales molestias dolorosas residuales, ya sea en la cicatriz palmar o en los pilares carpianos. ${ }^{5,6}$

Considerando las desventajas de la sección abierta del RF en el manejo quirúrgico del STC, dos artroscopistas, Okutsu y Chow, idearon y desarrollaron, de manera independiente y simultánea, dos técnicas diferentes de liberación endoscópica del túnel carpiano que tienen en común la sección ligamentaria infrarretinacular. ${ }^{7,8}$ De esta forma, surgieron las vigentes técnicas endoscópicas de uno y dos portales, que comparten complejidades procedimentales, pero logran resultados clínicos satisfactorios cuando los equipos quirúrgicos alcanzan un dominio técnico avanzado. ${ }^{11-16}$

Distintos grupos quirúrgicos han incorporado a la descompresión endoscópica del túnel carpiano como la técnica de elección, buscando obtener los resultados positivos que caracterizan a las técnicas quirúrgicas mininvasivas. No obstante, las ventajas comunes de los procedimientos endoscópicos de portal único y doble pueden verse francamente opacadas cuando surgen complicaciones neurovasculares graves. ${ }^{21-24}$

La técnica de doble portal de Chow para la sección endoscópica del RF es una opción fiable para el STC. Mediante la aplicación correcta del anestésico local, la localización precisa de los portales, la familiaridad con la anatomía endoscópica del carpo, la visualización clara de las estructuras del túnel carpiano y la destreza quirúrgica endoscópica es posible lograr resultados óptimos. La anestesia local permite que el paciente y el primer cirujano se puedan comunicar durante todo el procedimiento y así prevenir los potenciales riesgos intraoperatorios. ${ }^{17}$

Los resultados clínicos de este estudio de cohortes histórico que empleó la técnica de doble portal de Chow, durante una década, se comparan favorablemente con los publicados en estudios similares. En nuestra serie, se destacan el reducido número de complicaciones y los resultados satisfactorios en el 96\% de los casos, en la evaluación final. Cabe señalar que estas complicaciones ocurrieron en pacientes operados durante los primeros dos años de nuestra serie, y fueron siete casos de neuropraxia posquirúrgica transitoria, cuya recuperación se confirmó en el control de los 6 meses. ${ }^{11-16,25-29}$

Es importante destacar que las técnicas de liberación endoscópica de portal único y doble que se emplean en la actualidad tienen en común la característica de ser procedimientos quirúrgicos infrarretinaculares, con resultados clínicos comparables a largo plazo, por lo que la elección de una u otra técnica depende, más bien, de sutilezas procedimentales y afinidades instrumentales de los equipos quirúrgicos, que de sus ventajas y potenciales desventajas. $^{7-16,25-29}$

La desventaja común de las técnicas endoscópicas de uno o dos portales es la introducción de instrumental dentro de un túnel carpiano con sus estructuras anatómicas comprimidas, gesto que puede incrementar, en forma transitoria o permanente, el compromiso del nervio mediano. Por este motivo, han surgido estudios y experiencias clínicas que proponen el empleo de técnicas de liberación endoscópica suprarretinacular, que tienen como común denominador la sección del RF desde el exterior del túnel carpiano. No obstante, estos procedimientos endoscópicos pueden generar eventuales lesiones neurológicas en caso de variantes anatómicas, así como dañar mecanorreceptores en la región palmar de la muñeca, por lo que no parecen representar una alternativa superior frente a las vigentes técnicas infrarretinaculares. ${ }^{30-32}$

\section{CONCLUSIONES}

La retinaculotomía endoscópica del túnel carpiano mediante la técnica de dos portales de Chow es una intervención quirúrgica reglada, eficaz y segura para el tratamiento del STC. La tasa de complicaciones es mínima cuando la intervención está en manos de un equipo quirúrgico cohesionado, liderado por un primer cirujano experimentado. 
Conflicto de intereses: Los autores no declaran conflictos de intereses.

ORCID de Á. C. Ojeda: https://orcid.org/0000-0003-0136-5012

ORCID de V. A. Contreras: https://orcid.org/0000-0002-5269-0627

\section{BIBLIOGRAFÍA}

1. Atroshi I, Gummesson C, Johnsson R, Ornstein E, Ranstam J, Rosen I. Prevalence of carpal tunnel syndrome in a general population. JAMA 1999;282(2):153-8. https://doi.org/10.1001/jama.282.2.153

2. Paget J. Lectures on surgical pathology: Delivered at the Royal College of Surgeons of England. 2nd ed. Philadelphia: Lindsay \& Blakiston; 1854:42-3.

3. Learmonth JR. The principle of decompression in the treatment of certain diseases of peripheral nerves. Surg Clin North Am 1933;13:905-13.

4. Rodner CM, Katrincic J. Open carpal tunnel release. Tech Orthop 2006;21(1):3-11. https://doi.org/10.1097/01.bto.0000220086.17351.e2

5. Kluge W, Simpson R, Nicol A. Late complications after open carpal tunnel decompression. J Hand Surg Br 1996; 21(2):205-7. https://doi.org/10.1016/s0266-7681(96)80098-2

6. Boya H, Özcan Ö, Özteki N. Long-term complications of open carpal tunnel release. Muscle Nerve 2008;38(5): 1443-6. https://doi.org/10.1002/mus.21068

7. Okutsu I, Ninomiya S, Takatori Y, Ugawa Y. Endoscopic management of the carpal tunnel syndrome. Arthroscopy 1989;5(1):11-8. https://doi.org/10.1016/0749-8063(89)90084-4

8. Chow JC. Endoscopic release of the carpal ligament: a new technique for carpal tunnel syndrome. Arthroscopy 1989;5(1):19-24. https://doi.org/10.1016/0749-8063(89)90085-6

9. Agee JM, McCarroll HR, Tortosa RD, Berry DA, Szabo RM, Peimer CA. Endoscopic release of the carpal tunnel: a randomized prospective multicenter study. J Hand Surg Am 1992;17(6):987-95. https://doi.org/10.1016/s0363-5023(09)91044-9

10. Brown MG, Keyser B, Rothenberg ES. Endoscopic carpal tunnel release. J Hand Surg Am 1992;17(6):1009-11. https://doi.org/10.1016/S0363-5023(09)91047-4

11. Chow JC. The Chow technique of endoscopic release of the carpal ligament for carpal tunnel syndrome: four years of clinical results. Arthroscopy 1993;9(3):301-14. https://doi.org/10.1016/s0749-8063(05)80426-8

12. Atroshi I, Larsson GU, Ornstein E, Hofer M, Johnsson R, Ranstam J. Outcomes of endoscopic surgery compared with open surgery for carpal tunnel syndrome among employed patients: randomised controlled trial. $B M J$ 2006;24:332 (7556):1473. https://doi.org/10.1136/bmj.38863.632789.1F

13. Atroshi I, Hofer M, Larsson GU, Ornstein E, Johnsson R, Ranstam J. Open compared with 2-portal endoscopic carpal tunnel release: a 5-year follow-up of a randomized controlled trial. J Hand Surg Am 2009;34(2):266-72. https://doi.org/10.1016/j.jhsa.2008.10.026

14. Okutsu I, Hamanaka I, Yoshida A. Retrospective analysis of five-years and longer clinical and electrophysiological results of the world's first endoscopic management for carpal tunnel syndrome. Hand Surg 2013;18(3):317-23. https://doi.org/10.1142/S0218810413500330

15. Sayegh ET, Strauch RJ. Open versus endoscopic carpal tunnel release: a meta-analysis of randomized controlled trials. Clin Orthop Relat Res 2015;473(3):1120-32. https://doi.org/10.1007/s11999-014-3835-z

16. Argüelles S, Rodriguez M, Soutullo J, Santos G, Gelo M, Pereira E. Descompresión endoscópica del túnel carpiano. Resultados y complicaciones en 126 casos. Artroscopía 2018;25(4):131-5. https://www.revistaartroscopia.com.ar/ ediciones-anteriores/images/artroscopia/volumen-25-nro-4/25_04_05_Arguelles/25_04_Arguelles.pdf

17. Chow JCY, Papachristos AA. Endoscopic carpal tunnel release: Chow technique. Tech Orthop 2006;21(1):19-29. https://doi.org/10.1097/01.bto.0000221927.44752.6e

18. Sanz-Reig J, Lizaur-Utrilla A, Sanchez del Campo F, Maqueda-Abreu V. Estudio ecográfico del síndrome del túnel del carpo en la mujer. Rev Esp Cir Ortop Traumatol 2004;48(3):201-4.

https://doi.org/10.1016/S1888-4415(04)76197-4 
19. Levine DW, Simmons BP, Koris MJ, Daltroy LH, Hohl GG, Fossel AH, et al. A self-administered questionnaire for the assessment of severity of symptoms and functional status in carpal tunnel syndrome. J Bone Joint Surg Am 1993;75(11):1585-92. https://doi.org/10.2106/00004623-199311000-00002

20. Tulipan JE, Kim N, Ilyas AM, Matzon JL. Endoscopic carpal tunnel release with and without sedation. Plast Reconstr Surg 2018;141(3):685-690. https://doi.org/10.1097/PRS.0000000000004113

21. Nath RK, Mackinnon SE, Weeks PM. Ulnar nerve transection as a complication of two-portal endoscopic carpal tunnel release: a case report. J Hand Surg Am 1993;18(5):896-8. https://doi.org/10.1016/0363-5023(93)90063-9

22. Murphy RX, Jennings JF, Wukich DK. Major neurovascular complications of endoscopic carpal tunnel release. J Hand Surg Am 1994;19(1):114-8. https://doi.org/10.1016/0363-5023(94)90233-X

23. De Smet L, Fabry G. Transection of the motor branch of ulnar nerve as a complication of two-portal endoscopic carpal tunnel release: a case report. J Hand Surg Am 1995;20(1):18-9. https://doi.org/10.1016/s0363-5023(05)80050-4

24. Ryu SJ, Kim IS. Pseudoaneurysm of ulnar artery after endoscopic carpal tunnel release. J Korean Neurosurg Soc 2010;48(4):380-2. https://doi.org/10.3340/jkns.2010.48.4.380

25. Chow JC, Hantes ME. Endoscopic carpal tunnel release: thirteen years' experience with the Chow technique. J Hand Surg Am 2002;27(6):1011-8. https://doi.org/10.1053/jhsu.2002.35884

26. Oertel J, Schroeder HWS, Gaab MR. Dual-portal endoscopic release of the transverse ligament in carpal tunnel syndrome: result of 411 procedures with special reference to technique, efficacy, and complications. Neurosurgery 2006;59(2):333-40. https://doi.org/10.1227/01.NEU.0000223500.25131.99

27. Hankins CL, Brown MG, Lopez RA, Lee AK, Dang J, Harper RD. A 12-year experience using the Brown two-portal endoscopic procedure of transverse carpal release in 14.722 patients. Plast Reconstr Surg 2007;120(7):1911-21. https://doi.org/10.1097/01.prs.0000287287.85044.87

28. Uchiyama S, Nakamura K, Itsubo T, Murakami H, Hayashi M, Imaeda T, et al. Technical difficulties and their prediction in 2-portal endoscopic carpal tunnel release for idiopathic carpal tunnel syndrome. Arthroscopy 2013;29(5):860-9. https://doi.org/10.1016/j.arthro.2013.01.027

29. Vasiliadis HS, Georgoulas P, Shrier I, Salanti G, Scholten RJPM. Endoscopic release for carpal tunnel syndrome. Cochrane Database Sys Rev 2014;(1):CD008265. https://doi.org/10.1002/14651858.CD008265.pub2

30. Ip WJ, Sweed T, Fung KKB, Tipoe G, Pun TS. A new technique of single portal endoscopic carpal tunnel release. Tech Hand Up Extrem Surg 2012;16(1):27-9. https://doi.org/10.1097/BTH.0b013e318230db42

31. Ecker J, Perera N, Ebert J. Supraretinacular endoscopic carpal tunnel release: surgical technique with prospective case series. J Hand Surg Eur 2015;40(2):193-8. https://doi.org/10.1177/1753193414524688

32. Teh KK, Choo CH, Shanmugam R, Ngim JH, Ahmad TS. A novel supraretinacular endoscopic carpal tunnel release: Instrumentation and technique (cadaveric study). J Hand Surg Global Online 2019;1(4):222-5. https://doi.org/10.1016/j.jhsg.2019.07.003 